\title{
Uterine Imaging in Infertility and Assisted Reproduction
}

\author{
Akmal El-Mazny MD ${ }^{1,2 *}$, Mahmoud Edessy MD $^{2,3}$ \\ ${ }^{I}$ Department of Obstetrics and Gynecology, Faculty of Medicine, Cairo University, Egypt \\ ${ }^{2}$ Department of Obstetrics and Gynecology, Armed Forces College of Medicine (AFCM), Egypt \\ ${ }^{3}$ Department of Obstetrics and Gynecology, Faculty of Medicine, Al Azhar University, Egypt
}

*Corresponding Author: Akmal El-Mazny, MD, Department of Obstetrics and Gynecology, Faculty of Medicine, Cairo University, Egypt, Email: dr_akmalelmazny@yahoo.com

\begin{abstract}
Congenital uterine anomalies and acquired uterine lesions may affect uterine functions precluding successful pregnancy. Therefore, exclusion of any uterine pathology becomes an important step in infertility work-up. This review discusses the different techniques used for uterine imaging in infertility and assisted reproduction.
\end{abstract}

Keywords: Uterine imaging, Infertility, Assisted reproduction

\section{INTRODUCTION}

Despite advances in the field of assisted reproduction, only one-third of cycles started end in a pregnancy and one-fourth result in a live birth ${ }^{[1]}$. The uterus plays a major role in sperm migration, embryo implantation, and fetal development. Congenital uterine anomalies and acquired uterine lesions may affect such uterine functions precluding successful pregnancy.

Uterine pathologies are found to be present in $25 \%$ of infertile patients ${ }^{[2]}$. Structural abnormalities of the uterus, such as leiomyoma, endom etrial polyps, intrauterine adhesions (IUAs), adenomyosis and mullerian anomalies, may affect the reproductive outcome adversely, by interfering with implantation or causing spontaneous abortion. Therefore, exclusion of any uterine pathology becomes an important step in infertility work-up.

\section{DISCUSSION}

Uterine abnormalities may be visualized using variety of imaging techniques ${ }^{[3-5]}$ :

- Hysterosalpingography (HSG).

- Transvaginal sonography (TVS).

- Sonohysterography (SHG).

$-3 \mathrm{D} / 4 \mathrm{D}$ ultrasonography.

$-5 \mathrm{D}$ ultrasonography.

- Doppler ultrasonography.
- Magnetic resonance imaging (MRI).

- Endoscopic ultrasound.

\subsection{Hysterosalpingography (HSG)}

HSG is an indirect mean of showing the interior of the uterus. It has been the most commonly used technique in the evaluation of infertility. Endometrial polyps, fibroids, IUAs and müllerian anomalies are shown as filling defects or uterine cavity abnormalities.

These findings necessitate secondary investigation in the form of hysteroscopy to confirm and possibly treat the pathology. Compared with hysteroscopy, HSG is considered to have a relatively high false-positive and false-negative results ${ }^{[6-9]}$.

\subsection{Transvaginal Sonography (TVS)}

It has been used as a screening test for the assessment of uterine cavity and it is an integral part of IVF treatment. TVS is more specific (96.3\%) and sensitive (100\%) than HSG. In comparison with hysteroscopy, TVS has $84.5 \%$ sensitivity and $98.7 \%$ specificity ${ }^{[10]}$.

However, TVS may not distinguish between a hyperplasic endometrium and a large polyp, or differentiate between an arcuate and a septate uteri. Abnormal uterine findings on a baseline scan can be further evaluated with SHG.

\subsection{Sonohysterography (SHG)}

It improves the delineation of the uterine cavity and, therefore, could be an alternative to HSG 
and hysteroscopy as a screening test. Compared to hysteroscopy, SHG has $87.5 \%$ sensitivity, $100 \%$ specificity, $100 \%$ positive predictive value and $91.6 \%$ negative predictive value for the detection of uterine cavity abnormality ${ }^{[11]}$.

\subsubsection{D/4D Ultrasonography}

The advent of transvaginal 3D/4D ultrasonography has enabled the accurate, noninvasive, outpatient diagnosis of congenital uterine anomalies. It has also enabled large-scale screening and morphological analysis of mullerian anomalies.

Some studies reported the value of 3D/4Dguided embryo transfer in localization of the maximal implantation potential (MIP) point compared to the traditional 2D ultrasound ${ }^{[12]}$. The MIP varies from patient to patient dependent upon the shape of the uterine cavity.

Compared to hysteroscopy, 3D SHG reached a sensitivity of $94.2 \%$ and specificity of $98.5 \%$, with a positive predictive value of $98.0 \%$ and a negative predictive value of $95.7 \%{ }^{[13]}$.

\subsubsection{Ultrasonography}

The state of the art machine differs from the 3D/4D machines in making highly precise calculations automatically. A special feature of the machine is that the $3 \mathrm{D}$ information is digitalized in the form of 'tissue-blocks' which then can be stored and transferred.

It features the latest elastography that makes it easier for users to distinguish benign from malignant masses through acquiring the strain ratio between the target and reference area faster than the previous models. This means that it could identify the isoechoic lesions that were missed by 3D ultrasound.

\subsection{Doppler Ultrasonography}

In addition to the ability to detect some of the intrauterine lesions as the feeding vessels of polyps and the depth of myoma, Doppler ultrasonography can be utilized to assess the endometrial receptivity. 3D power Doppler is used for determination of endometrial and subendometrial blood flow which affects embryo transfer and implantation ${ }^{[14,15]}$.

Ultrasonographic and Doppler criteria of good endometrial receptivity:

- Endometrial thickness $>7 \mathrm{~mm}$.

- Hypoechogenic endometrium with 3 well delineated layers.

- Endometrial volume $>2 \mathrm{ml}$.

- Uterine PI < 3.

- Presence of subendometrial vascular flow.
- High VI, FI and VFI in endometrial and subendometrial zone.

\subsection{Magnetic Resonance Imaging (MRI)}

MRI is especially accurate in the diagnosis of congenital uterine anomalies; it is noninvasive and avoids the risk of irradiation. Its main disadvantages include high cost and limited availability; thus, its role in the evaluation of uterine cavity prior to IVF remains minor.

\subsection{Endoscopic Ultrasound}

Intraoperative ultrasound has gained an established role in many surgical procedures. It has been introduced mainly to overcome the two major drawbacks of endoscopy: the ability to show only the surface of the organs and the lack of manual palpation of the structures.

Hysteroscopic 5D ultrasound can be used during the resection of IUAs and uterine septa, hysteroscopic myomectomy, and for differentiation between septate and bicornuate uteri. Robotic 5D ultrasound is the latest version of intraoperative sonography. It can accurately identify and track the target tissue during the surgical procedures.

\section{CONCLUSION}

Structural abnormalities of the uterus, such as leiomyoma, endometrial polyps, IUAs, adenomyosis and mullerian anomalies, may affect the reproductive outcome adversely, by interfering with implantation or causing spontaneous abortion. Uterine abnormalities may be visualized using variety of imaging techniques including HSG, TVS, SHG, 3D/4D ultrasonography, Doppler ultrasonography, and MRI. Other potential techniques, such as 5D ultrasonography and endoscopic ultrasound, remain under trial.

\section{REFERENCES}

[1] Society for Assisted Reproductive Technology (SART); American Society for Reproductive Medicine (ASRM). Assisted reproductive technology in the United States: 2001 results generated from the ASRM/SART registry. Fertil Steril. 2007; 87: 1253-66.

[2] Levi Setti PE, Colombo GV, Savasi V, et al. Implantation failure in assisted reproduction technology and a critical approach to treatment. Ann N Y Acad Sci. 2004; 1034: 184-99.

[3] Loverro G, Nappi L, Vicino M, et al. Uterine cavity assessment in infertile women: comparison of transvaginal sonography and hysteroscopy. Eur J Obstet Gynecol Reprod Biol. 2001; 100: 67-71. 
[4] Ragni G, Diaferia D, Vegetti W, et al. Effectiveness of sonohysterography in infertile patient work-up: a comparison with transvaginal ultrasonography and hysteroscopy. Gynecol Obstet Invest. 2005; 59: 184-8.

[5] Devroey P, Fauser BC, Diedrich K; Evian Annual Reproduction (EVAR) Workshop Group 2008. Approaches to improve the diagnosis and management of infertility. Hum Reprod Update. 2009; 15: 391-408.

[6] Preutthipan S, Linasmita V. A prospective comparative study between hysterosalpingography and hysteroscopy in the detection of intrauterine pathology in patients with infe rtility. J Obstet Gynaecol Res. 2003; 29:33-7.

[7] Roma Dalfó A, Ubeda B, Ubeda A, et al. Diagnostic value of hysterosalpingography in the detection of intrauterine abnormalities: a comparison with hysteroscopy. Am J Roentgenol. 2004; 183: 1405-9.

[8] Ludwin A, Ludwin I, Banas T, et al. Diagnostic accuracy of sonohysterography, hysterosalpingography and diagnostic hysteroscopy in diagnosis of arcuate, septate and bicornuate uterus. J Obstet Gynaecol Res. 2011; 37:17886.

[9] Vujović M, Garalejic E, Arsic B, et al. Hysterosalpingography versus hysterscopy in intrauterine pathology research of infertile patients. Clin Exp Obstet Gynecol. 2015; 42:141-5.
[10] Shalev J, Meizner I, Bar-Hava I, et al. Predictive value of transvaginal sonography performed before routine diagnostic hysteroscopy for evaluation of infertility. Fertil Steril. 2000; 73: 412-7.

[11] Seshadri S, El-Toukhy T, Douiri A, et al. Diagnostic accuracy of saline infusion sonography in the evaluation of uterine cavity abnormalities prior to assisted reproductive techniques: a systematic review and metaanalyses. Hum Reprod Update. 2015; 21:26274.

[12] Gergely RZ, DeUgarte CM, Danzer H, et al. Three dimensional/four dimensional ultrasound guided embryo transfer using maximal implantation potential point. Fertil Steril. 2005; 84: 500-3.

[13] El-Sherbiny W, El-Mazny A, Abou-Salem N, et al. The diagnostic accuracy of two- vs three dimensional sonohysterography for evaluation of the uterine cavity in the reproductive age. $\mathbf{J}$ Minim Invasive Gynecol. 2015;22:127-31

[14] El-Mazny A, Abou-Salem N, Elshenoufy H. Doppler study of uterine hemodynamics in women with unexplained infertility. Eur $\mathbf{J}$ Obstet Gynecol Reprod Biol. 2013; 171:84-7.

[15] El-Mazny A, Ramadan W, Kamel A, et al. Effect of hydrosalpinx on uterine and ovarian hemodynamics in women with tubal factor infertility. Eur J Obstet Gynecol Reprod Biol. 2016; 199:55-9.

Citation: Akmal El-Mazny, Mahmoud Edessy. Uterine Imaging in Infertility and Assisted Reproduction. ARC Journal of Gynecology and Obstetrics. 2017; 2(1):1-3. doi:dx.doi.org/10.20431/2456-0561.0201001.

Copyright: (C) 2017 Authors. This is an open-access article distributed under the terms of the Creative Commons Attribution License, which permits unrestricted use, distribution, and reproduction in any medium, provided the original author and source are credited. 